\title{
PRODUTIVIDADE DA SOJ A EM RESPOSTA À APLICAÇÃO DE MOLIBDÊNIO E INOCULAÇÃO COM Bradyrhizobium japonicum ${ }^{(1)}$
}

\author{
Eloir Paulo Gris ${ }^{(2)}$, Ana Maria Conte e Castro(3) \\ \& Fábio Faria de Oliveira(4)
}

\begin{abstract}
RESUMO
A soja em simbiose com Bradyrhizobium japonicum é capaz de ter a sua exigência de $\mathbf{N}$ satisfeita com a fixação biológica de $\mathbf{N}_{2}$ (FBN). Entretanto, a FBN é afetada pela deficiência Mo, visto que este nutriente faz parte da enzima nitrogenase responsável pelo processo. Foi realizado um experimento, em condição de campo, em Latossolo Vermelho eutroférrico, no município de Palotina, PR, com o objetivo de avaliar o tratamento de sementes com Mo, inoculação de B. japonicum e adubação foliar de Mo na produtividade da soja. Os tratamentos foram quatro doses de $M o\left(0,40,80\right.$ e $\left.160 \mathrm{~g} \mathrm{ha}^{-1}\right)$ aplicadas em adubação foliar e tratamento com aplicação nas sementes de $40 \mathrm{~g} \mathrm{ha}^{-1} \mathrm{de} \mathrm{Mo}$, combinados com e sem inoculação com B. japonicum. Não foram observados efeitos estatí́sticos significativos do tratamento de sementes com Mo, inoculação de B. japoni cum e adubação foliar de Mo na produtividade da soja. Não foram obtidas diferenças significativas entre os tratamentos, obtendo-se tendência apra ganho de produtividade apenas com aplicação foliar de $80 \mathrm{~g} \mathrm{ha}^{-1}$ de Mo e tratamento de sementes com $40 \mathrm{~g} \mathrm{ha}^{-1}$.
\end{abstract}

Termos de indexação: adubação foliar, tratamento de sementes, micronutriente, rizóbio.

\footnotetext{
(1) Recebido para publicação em outubro de 2001 e aprovado em novembro de 2004.

(2) Engenheiro-Agrônomo da Cooperativa Agrícola Mista do Oeste do Paraná. Rua Paraíba 1635, CE P 85960-000 Marechal Cândido Rondon (PR).

(3) Professora de Agronomia da Universidade Estadual do Oeste do Paraná - UNIOESTE. Rua Pernambuco 1777, CEP 85960-000 Marechal Cândido Rondon (PR). E-mail: acastro@unioeste.br

(4) Acadêmico do Curso de Agronomia, UNIOESTE.
} 


\title{
SUMMARY: SOYBEAN YIELD IN RESPONSE TO MOLYBDENUM AND Bradyrhizobium japonicum I NOCULATION
}

\begin{abstract}
Soybean in symbiosis with Bradyrhizobium japonicum is able to satisfy its nitrogen $\left(\mathrm{N}_{2}\right)$ demand with biological nitrogen fixation (BNF). However, BNF can be affected by molybdenium deficiency because this micronutrient is part of the nitrogenase enzyme responsiblefor the process. An experiment was conducted under fiedd conditions on a Red L atosol (Oxisol) in Palotina, stateof Paraná, Brazil. Theeffects of seed treatments with Mo, $B$. japonicum inoculation as well as fol iar Mo fertilizati on on soybean yield wereevaluated. Four Morates $\left(0,40,80\right.$ and $\left.160 \mathrm{~g} \mathrm{ha}^{-1}\right)$ wereapplied as foliar fertilizer in two Moapplication forms (without Mo, seed treatment with Mo $40 \mathrm{~g} \mathrm{ha}^{-1}$ ) combined with and without $\mathrm{B}$. japonicum inoculation. There were no si gnificant effects of the seed treatments with Mo, inoculation with B. japonicum and fol iar Mo fertilization on soybean yield were observed. The economic analysis showed negative effects; only when $80 \mathrm{~g} \mathrm{ha}^{-1}$ were applied as fol iar fertilization and the seed treatment with $40 \mathrm{~g} \mathrm{ha}^{-1}$ an economic yield was obtained.
\end{abstract}

Index terms: fol iar fertilization, seed treatments, mi cronutrient, rhizobion.

\section{INTRODUÇÃO}

A soja, quando em simbiose com bactérias do gênero Bradyrhizobium, é capaz de ter a sua exigência de $\mathrm{N}$ totalmente satisfeita com o processo de fixação biológica de $\mathrm{N}_{2}$ (Mengel \& Kirkby, 1987). Entretanto, a fixação biológica éseriamente afetada, quando ocorre deficiência de Mo, tendo em vista que este nutriente faz parte da enzima nitrogenase responsável pelo processo da fixação (Marschner, 1986).

Altas produtividades de soja são verificadas com a ocorrência do processo de fixação biológica de $\mathrm{N}_{2}$, em sol os com condições ótimas de fertilidade. Nesse contexto, é marcante a influência do Mo que atua também na redutase do nitrato, responsável pela redução do $\mathrm{NO}_{3}{ }^{-}$para ser assimilado pela planta (Marschner, 1986). Assim, a deficiência de Mo poderia ser uma das causas da redução da produtividade observada em al gumas áreas no oeste do estado do Paraná.

Como as quantidades de Mo requeridas pelas plantas são pequenas, a sua aplicação via semente constitui a forma mais prática e eficaz de seu suprimento (Gupta \& Lipsett; Reisenauer, citados por Vidor \& Peres, 1988). Não há indicações de que haja toxidez ao Bradyrhizobium, quando a peletização com baixas doses de Mo é feita imediatamente antes da semeadura da soja. Neste caso, ocorrem uma excel ente nodulação e aumento na velocidade de crescimento, no número de sementes por vagem e no rendimento de grãos (Sfredo et al., 1997; Rosolem, 1982).

Apesar de não serem totalmente conhecidas a extensão e a importância da deficiência de Mo na produtividade da soja, o problema existe (Sfredo et al., 1997) e tenderá a se agravar à medida que se intensificar o cultivo de soja pel o uso de variedades altamente produtivas, técnicas de manejo voltadas para alta produtividade e solos com restrições químicas crescentes.

A aplicação demi cronutrientes visandoà correção de deficiências nutricionais pode ser feita de três modos: diretamente no solo junto com a adubação convencional (Cheng, 1955), em aplicação foliar (Conte\& Castro, 1991) e via tratamento desementes (Cheng, 1985; Parducci et al., 1989). Como as quantidades de Mo requeridas pelas plantas são bastante reduzidas, sua aplicação, juntamente com o tratamento de sementes mais fungicidas, constitui a forma mais prática, eficiente e econômica de correção da deficiência (Cheng, 1985; Vidor \& Peres, 1988; Parducci et al., 1989).

A calagem, de maneira geral, aumenta a disponibilidade de Mo (Malavolta et al., 1997), e a maior disponibilidade ocorre em $\mathrm{pH}$ superior a 7 (Gupta \& Lipsett, 1981; Horowitz, 1978). A possibilidade de ocorrer deficiência de Mo é maior em sol os de baixa fertilidadee com pH ácido, podendo a calagem corrigir essa deficiência, desde que o solo tenha teores adequados deste micronutriente, considerando quea atividade do mol ibdato aumenta cem vezes para cada unidade de aumento de $\mathrm{pH}$ (Lindsay, 1979).

Conforme Borkert (1987), a utilização de pulverizações de Mo em soja não tem surtido efeito no aumento do rendimento de grãos. Essa falta de resposta talvez possa ser explicada pelas baixas concentrações dos nutrientes nas misturas de pulverizações, que não são suficientes para influenciar o rendimento da cultura, já que altas concentrações podem provocar queima de fol has. 
As formas de Mo mais utilizadas em adubações são os molibdatos de $\mathrm{Na}$ e de amônio e o trióxido de Mo, sendo também utilizados o ácido molíbdico e fertilizantes compostos que contêm o Mo em sua composição como as "fritas" - FTE (fritted trace elements). Essas formas podem ser fornecidas às plantas como adubo de solo, aspersão foliar (exceto o FTE) ou peletizadas com as sementes (Vidor \& Peres, 1988).

As respostas à adubação com Mo, no Brasil, têm sido variáveis. Diversos experimentos, realizados com soja, não apresentaram aumentos de rendimento de grãos ou matéria seca, (Kolling et al., 1981; LámSanchez \& Awad, 1976; Mascarenhas et al., 1967, 1973). Entretanto, aumentos significativos foram obtidos por Bellintani Neto \& Lám-Sanchez (1974) e Buzetti et al. (1981), em resposta à adubação com $400 \mathrm{~g} \mathrm{ha}^{-1}$ de molibdato de $\mathrm{Na}$, em Latossolo Vermelho-Escuro. Similarmente, Vitti et al. (1984) obtiveram aumentos de até 32,7 \% pela utilização de doses crescentes de um produto comercial que continha $10 \%$ de Mo e $1 \%$ de Co.

Pela aplicação foliar de Mo, também tem sido possível aumentar a produtividade do feijoeiro (J acob-Neto \& Franco, 1986) e da soja (J acob-Neto \& Franco, 1995; citados por Amorim et al., 1997).

A partir dessas considerações, realizou-se um experimento, em condi ções de campo, com o objetivo de avaliar o efeito da aplicação de diferentes concentrações de Mo, via tratamento de sementes e adubação foliar, e da inoculação das sementes com inoculante comercial de Bradyrhizobium japonicum sobre a produtividade da soja, em condições de plantio direto.

\section{MATERIAL E MÉTODOS}

O experimento foi realizado no campo, no município dePalotina (PR), em solo classificado como Latossolo Vermelho eutroférrico textura argilosa (E mbrapa, 1999), cujas características químicas foram: Matéria orgânica $\left(\mathrm{g} \mathrm{dm}^{-3}\right)=25,0 ; \mathrm{pH}$ em água $(1: 2,5)=5,3 ; \mathrm{P}-$ Resina $\left(\mathrm{mg} \mathrm{dm}^{-3}\right)=32,0 ; \mathrm{P}-$ Mehlich $\left(\mathrm{mg} \mathrm{dm}^{-3}\right)=15,0 ; \mathrm{K}\left(\mathrm{cmol}_{\mathrm{c}} \mathrm{dm}^{-3}\right)=0,31 ; \mathrm{Al}^{3+}$ $\left(\mathrm{cmol}_{\mathrm{C}} \mathrm{dm}^{-3}\right)=0,20 ; \mathrm{Ca}^{2+}\left(\mathrm{cmol}_{\mathrm{c}} \mathrm{dm}^{-3}\right)=3,10 ; \mathrm{Mg}^{2+}$ $\left(\mathrm{cmol}_{\mathrm{C}} \mathrm{dm}^{-3}\right)=1,40 ; \mathrm{S}\left(\mathrm{mg} \mathrm{dm}^{-3}\right)=2,2 ; \mathrm{H}+\mathrm{Al}$ $\left(\mathrm{cmol}_{\mathrm{C}} \mathrm{dm}^{-3}\right)=3,80$; SB $\left(\mathrm{cmol}_{C} \mathrm{dm}^{-3}\right)=4,81$; CTC total $\left(\mathrm{cmol}_{\mathrm{C}} \mathrm{dm}^{-3}\right)=8,61 ; \mathrm{V}(\%)=56 ; \mathrm{B}\left(\mathrm{mg} \mathrm{dm}^{-3}\right)=0,1$; Fe $\left(\mathrm{mg} \mathrm{dm}^{-3}\right)=83,5 ; \mathrm{Mn}\left(\mathrm{mg} \mathrm{dm}^{-3}\right)=41,8 ; \mathrm{Cu}$ $\left(\mathrm{mg} \mathrm{dm}^{-3}\right)=2,9$ e Zn $\left(\mathrm{mg} \mathrm{dm}^{-3}\right)=1,1$.

Foram utilizadas sementes de soja, cultivar BR16, recomendadas para a região (E mbrapa, 1997). Os tratamentos foram arranjados no esquema fatorial $2 \times 4+2$, ou seja com e sem inoculação das sementes com Bradyrhizobium japonicum, quatro doses de Mo aplicada via foliar 25 dias após a emergência $\left(0,40,80\right.$ e $\left.160 \mathrm{~g} \mathrm{ha}^{-1}\right)$ e dois tratamentos adicionais, que consistiram do tratamento das sementes com Mo (40 g ha-1), com e sem inoculação das sementes, num total de 10 tratamentos.

O delineamento experimental utilizado foi o de blocos inteiramente casualizados com quatro repetições. Quando programado, foi utilizado o inoculante comercial Nitral turfa de Bradyrhizobium japonicum, na dose de $200 \mathrm{~g}$ por $50 \mathrm{~kg}$ sementes. Para a aplicação de Mo via foliar ou nas sementes, quando programada, foi utilizado molibdato de amônio. Para o tratamento das sementes, foi utilizada a dose de 0,3 $\mathrm{g}$ de Mo para $0,5 \mathrm{~kg}$ de sementes (Quadro 1).

As parcelas foram constituídas de quatro linhas de 6,0 m de comprimento com espaçamento entre linha de 0,5 m, com densidade de semeadura de 18 sementes por metro linear. Para obter a área útil da parcela, foram utilizadas as duas linhas centrais, eliminando-se 0,5 m decada extremidade.

As adubações foram efetuadas no sulco de semeadura na dose de $160 \mathrm{~g} \mathrm{ha}^{-1}$ da fórmula comercial 0-30-10, segundo análise de solo, e as adubações foliares foram realizadas no período da manhã, entre 8 e $9 \mathrm{~h}$, e ovol ume da calda aplicada foi de $200 \mathrm{~L} \mathrm{ha}^{-1}$, com pulverizador costal tipo J acto (20 L).

O experimento foi instalado dentro de uma lavoura comercial sob plantio direto na palha (oito anos) e utilizou-se a rotação de culturas (soja, nabo, milho, aveia) na safra agrícola de 1997/1998.

As vagens foram col hi das manualmentee levadas ao terreiro para a batedura e, quando secas, foram, posteriormente separados os grãos e a pal hada.

Os dados de rendimento de grãos foram submetidos à análise de variância e as médias comparadas pel o teste de Tukey a $5 \%$, pelo programa Estat.

Para a anál ise econômica, foram cotados os preços dos produtos comerciais utilizados no experimento e o preço da saca de soja e inoculante comercial à base de turfa, conforme COOPERVALE (06/10/ 2000), e da utilização do molibdato de amônio.

\section{RESULTADOS E DISCUSSÃO}

Não houve diferença estatística entre os tratamentos quanto à produtividade (Quadro 1). Esses resultados discordam dos da Embrapa (1999a), porém estão de acordo com os de Pessoa et al. (1999).

Mesmo não ocorrendo efeitos significativos com a aplicação de Mo, a produtividade da soja aumentou de $2.219 \mathrm{~kg} \mathrm{ha}^{-1}$, no tratamento testemunha, para $2.413 \mathrm{~kg} \mathrm{ha}^{-1}$, com a aplicação de $40 \mathrm{~g} \mathrm{ha}^{-1}$ de Mo via pel etização das sementes, e para $2.415 \mathrm{~kg} \mathrm{ha}^{-1}$, com a adubação foliar de $80 \mathrm{~g} \mathrm{ha}^{-1}$ de $\mathrm{Mo}$, aumentando, portanto, em $196 \mathrm{~kg} \mathrm{ha}^{-1}$ a produção de grãos com esta adubação foliar. 


\section{Quadro 1. Resultados médios de produtividade da soja, para diferentes tratamentos}

\section{Tratamento}

$\begin{array}{lc}\text { Testemunha } & 2.219 \mathrm{a} \\ \text { Inoculante Nitral turfa } & 1.692 \mathrm{a} \\ \text { Mo foliar - } 40 \mathrm{~g} \mathrm{ha}^{-1} \mathrm{~S} / \mathrm{I} & 2.085 \mathrm{a} \\ \text { Mo foliar - } 40 \mathrm{~g} \mathrm{ha}^{-1} \mathrm{C} / \mathrm{I} & 1.952 \mathrm{a} \\ \text { Mo foliar - } 80 \mathrm{~g} \mathrm{ha}^{-1} \mathrm{~S} / \mathrm{l} & 2.415 \mathrm{a} \\ \text { Mo foliar - } 80 \mathrm{~g} \mathrm{ha}^{-1} \mathrm{C} / \mathrm{I} & 2.179 \mathrm{a} \\ \text { Mo foliar - } 160 \mathrm{~g} \mathrm{ha}^{-1} \mathrm{~S} / \mathrm{I} & 1.966 \mathrm{a} \\ \text { Mo foliar - } 160 \mathrm{~g} \mathrm{ha}^{-1} \mathrm{C} / \mathrm{I} & 1.945 \mathrm{a} \\ \text { Mo semente - } 40 \mathrm{~g} \mathrm{ha}^{-1} \mathrm{C} / \mathrm{I} & 2.177 \mathrm{a} \\ \text { Mo semente - } 40 \mathrm{~g} \mathrm{ha}^{-1} \mathrm{~S} / \mathrm{I} & 2.413 \mathrm{a} \\ \text { Média } & 2.104 \\ \text { C.V. (\%) } & 17,25 \\ \text { F } & 0,63\end{array}$

Médias seguidas pela mesma letra não diferem a 5 \% testeTukey.

A ausência de resposta à adição de Mo pode estar relacionada com níveis adequados de disponibilidade de Mo no solo ou com concentrações de Mo na semente suficiente para satisfazer as necessidades das plantas (I shizuka, 1982).

Em relação à falta de diferença significativa entre sementes não tratadas eas tratadas com inoculante comercial é provável que o solo já disponha de populações estabelecidas de bactérias que proporcionaram boa nodulação e fixação biológica do $\mathrm{N}_{2}$, considerando que, nesse local, vem sendo cultivada a soja nos últimos oito anos em sistema de plantio direto e onde foram realizadas inoculações do rizóbio.

Altas concentrações de molibdato de amônio (160 $\mathrm{g} \mathrm{ha}^{-1}$ ), via foliar, podem ter provocado efeito tóxico às plantas, apresentando, portanto, produção menor do que a testemunha.

Para respostas à aplicação do Mo e para futuras recomendações desse micronutriente via foliar e, ou, tratamento de sementes, o experimento deve ser repetido em outras classes de solos do Oeste do Paraná e, principalmente, em outro sistema de cultivo.

\section{CONCLUSÕES}

1. Adubações fol iares e tratamentos de sementes com produtos à base de M o não foram eficientes em aumentar a produtividade da soja.

2. O uso de inoculante comercial de rizóbio não foi eficiente no aumento da produção da soja.

\section{AGRADE CIMENTO}

Homenagem póstuma a Antonio Carlos dos Santos Pessoa.

\section{LITE RATURA CITADA}

AMORIM, R.S.S.; TAKETA, S.T.; SENNA, L.F.N.; DORNELES, M.S. \& J ACOB-NETO, J. Efeito da aplicação foliar de sal de cozinha e Mo na nodulação da soja. In.: CONGRESSO BRASILEIRO DE CIÊNCIA DO SOLO, Rio de J aneiro, 1997. Anais. Rio de J aneiro, 1997. CD-ROM

BELLINTANI NETO, A.M. \& LAM-SÁNCHEZ, A. Efeito de Mo sobre a nodulação e produção de soja (Glycine max (L.) Meril ). Científica, 1:13-17, 1974.

BORKERT, C.M. Soja: adubação foliar. Londrina, Embrapa Centro Nacional de Pesquisa da Soja, 1987. 34p.

BUZETTI, S.; MAURO, A.O. \& VARGAS, J .T.D. Efeito de vários micronutrientes na cultura de soja. In: UNIVERSIDADE ESTADUAL PAULISTA. Relatório técnico-científico. IIha Solteira, 1981. p.66-68.

CHENG, T. The effect of seed treatment with microelements upon the germination and early growth of wheat. Sci. Sinica, 4:129-135, 1955.

CONTE E CASTRO, A.M. Adubação foliar e tratamento de sementes de feijoeiro com nutrientes, vitamina $B_{1} e$ metionina. Botucatu, Universidade Estadual de São Paulo, 1991. $97 p$

EMPRESA BRASILEIRA DE PESQUISA AGROPECUÁRIA EMBRAPA. Sistema Brasileiro de Classificação de Solos. Brasília, 1999 b. 412 p.

EMPRESA BRASILEIRA DE PESQUISA AGROPECUÁRIA EMBRAPA. Centro Nacional de Pesquisa de Solos. Sistema brasileiro de classificação de solo: 4a aproximação. Rio de J aneiro, 1997. 169p.

EMPRESA BRASILEIRA DE PESQUISA AGROPECUÁRIA EMBRAPA. Centro Nacional de Pesquisa de Soja. Recomendações técnicas para a cultura da soja no Paraná 1999/2000. Londrina, 1999a. 236p.

GUPTA, U.C. \& LIPSETT, J. Molybdenum in soil, plants and animals. Adv. Agron., 34:73-115, 1981.

HOROWITZ, A. Os íons do Mo no sol o: um exemplo da aplicação dos diagramas EH-pH. R. Bras. Ci. Solo, 2:98-103, 1978.

ISHIZUKA, J . Characterization of molybdenum absorption and translocation in soybean plants. Soil Sci. Plant Nutr., 28:6378, 1982.

KOLLING, J .; SCHOLLES, D. \& BROSE, A. Efeito do Mo aplicado em diferentes formulações sobre a nodulação e rendimentos de grãos de soja (Glycine max (L.) Merril). Agron. Sulriogr., 17:239-248, 1981.

LAM-SÁNCHEZ, A. \& AWAD, M. Efeito da simazina e do Mo no rendimento, conteúdo protéico e nodulação da soja e da simazina no rendimento e conteúdo protéico do feijoeiro (Phaseol us vulgaris L.). Científica, 4:56-58, 1976. 
LINDSAY, W.L. Chemical equilibria in soils. New York: J ohn Wiley, 1979. 449p.

MALAVOLTA, E.; VITTI, G.C. \& OLIVEIRA, S.A. Avaliação do estado nutricional das plantas: princípios e aplicações. Piracicaba, Potafos, 1997. 319p.

MARSCHNER, H. Mineral nutrition of higher plants. London, Academic Press, 1986. 674p.

MASCARENHAS, H.A.A.; MIYASAKA, S.; FREIRE, E.S. \& IGUE, T. Adubação da soja. VI - Efeitos do enxofre e de vários micronutrientes ( $\mathrm{Zn}, \mathrm{Cu}, \mathrm{B}, \mathrm{Mn}, \mathrm{Fe}$ e Mo) em Latossolo Roxo com vegetação de Cerrado. Bragantina, 26:373-379, 1967.

MASCARENHAS, H.A.A.; KIIHL, R.A.S.; NAGAI, V.\& BATAGLIA, O.C. Aplicação de micronutrientes em soja cultivada em sol os de Cerrado. O Agronômico, 25:71-73, 1973.

MENGEL, K. \& KIRKBY, E.A. Principles of plant nutrition. Bern, International Potash Institute, 1987. 667p.

PARDUCCI, S.; SANTOS, O.S. \& CAMARGO, R.P. Micronutrientes Biocrop. Campinas, Microquímica, 1989. 101p.
PESSOA, A.C.S.; LUCHESE, E.B.; CAVALLET, L.E. \& GRIS, E.P. Produtividade de soja em resposta à adubação foliar, tratamento de sementes com molibdênio e inoculação com Bradirhizobiumjaponicum. Maringá- PR. Acta Scientiarum, 21:531-535, 1999

ROSOLEM, C.A. Nutrição mineral e adubação da soja. 2.ed. Botucatu, UNESP, 1982. p.22-23. (Boletim Técnico, 6)

SFREDO, G.J .; BORKERT, C.M.; NEPOMUCENO, A.L. \& OLIVEIRA, M.C.N. Eficácia de produtos contendo micronutrientes, aplicados via semente, sobre produtividade e teores de proteína da soja. R. Bras. Ci. Solo, 21:41-45, 1997.

VIDOR, C. \& PERES, J.R.R. Nutrição das plantas com molibdênio e cobalto. In: BORKERT, C.M. \& LANTMANN, A.F., eds. Enxofre e micronutrientes na agricultura brasileira. Londrina: Embrapa/CNPSo/SBCS, 1988. p.179204.

VITTI, G.L.;FORNASIER, D. \& PEDROSO, P.A.C. Fertilizantes com molibdênio e cobalto na cultura da soja. R. Bras. Ci. Solo, 8:349-352, 1984. 